\title{
AFRL-RI-RS-TR-2015-036
}

\section{COMPUTATIONAL HOMOLOGY FOR SOFTWARE VALIDATION}

SYRACUSE UNIVERSITY

MARCH 2015

FINAL TECHNICAL REPORT

STINFO COPY

\section{AIR FORCE RESEARCH LABORATORY INFORMATION DIRECTORATE}




\section{NOTICE AND SIGNATURE PAGE}

Using Government drawings, specifications, or other data included in this document for any purpose other than Government procurement does not in any way obligate the U.S. Government. The fact that the Government formulated or supplied the drawings, specifications, or other data does not license the holder or any other person or corporation; or convey any rights or permission to manufacture, use, or sell any patented invention that may relate to them.

This report is the result of contracted fundamental research deemed exempt from public affairs security and policy review in accordance with SAF/AQR memorandum dated 10 Dec 08 and AFRL/CA policy clarification memorandum dated 16 Jan 09. This report is available to the general public, including foreign nationals. Copies may be obtained from the Defense Technical Information Center (DTIC) (http://www.dtic.mil).

AFRL-RI-RS-TR-2015-036 HAS BEEN REVIEWED AND IS APPROVED FOR PUBLICATION IN ACCORDANCE WITH ASSIGNED DISTRIBUTION STATEMENT.

FOR THE DIRECTOR:

/ S /

PAUL ALSING

Work Unit Manager
/ S /

MARK H. LINDERMAN

Technical Advisor, Computing

\& Communications Division

Information Directorate

This report is published in the interest of scientific and technical information exchange, and its publication does not constitute the Government's approval or disapproval of its ideas or findings. 


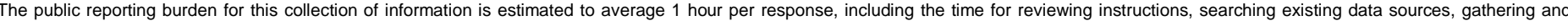

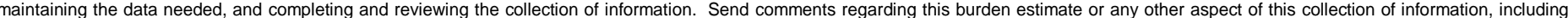

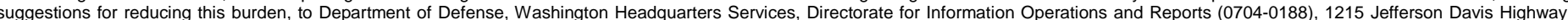

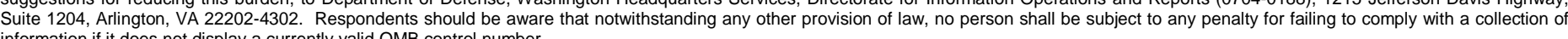

information if it does not display a currently valid OMB control number.
PLEASE DO NOT RETURN YOUR FORM TO THE ABOVE ADDRESS

\begin{tabular}{|c|c|r}
\hline $\begin{array}{c}\text { 1. REPORT DATE }(D D-M M-Y Y Y Y) \\
\text { MARCH } 2015\end{array}$ & 2. REPORT TYPE & 3. DATES COVERED (FrOm - To) \\
FINAL TECHNICAL REPORT & SEP 2011 - SEP 2014
\end{tabular}

\section{TITLE AND SUBTITLE}

COMPUTATIONAL HOMOLOGY FOR SOFTWARE VALIDATION

\section{5a. CONTRACT NUMBER}

FA8750-11-2-0275

N/A

5c. PROGRAM ELEMENT NUMBER

$65502 \mathrm{D}$

6. AUTHOR(S)

Howard A. Blair 5d. PROJECT NUMBER

T2GT

5e. TASK NUMBER

US

5f. WORK UNIT NUMBER

YR
7. PERFORMING ORGANIZATION NAME(S) AND ADDRESS(ES)

Dept. of Elec Eng \& Comp Sci, Syracuse University

4-206 Center for Sci \& Tech

Syracuse, NY 13244-4100

\section{SPONSORING/MONITORING AGENCY NAME(S) AND ADDRESS(ES)}

Air Force Research Laboratory/RITA

525 Brooks Road

Rome NY 13441-4505
8. PERFORMING ORGANIZATION REPORT NUMBER

\section{DISTRIBUTION AVAILABILITY STATEMENT}

Approved for Public Release; Distribution Unlimited. This report is the result of contracted fundamental research deemed exempt from public affairs security and policy review in accordance with SAF/AQR memorandum dated 10 Dec 08 and AFRL/CA policy clarification memorandum dated 16 Jan 09.

\section{SUPPLEMENTARY NOTES}

\section{ABSTRACT}

The research objective, to develop new objective tools to aid in software verification and validation based on topological methods, found that the application of topology to software with compound non-numerical, non-metric data-types, proved to be too course-grained to provide a mathematical foundation for rigorously reasoning about the evolution of the compound state of executing software and overcame this difficult by applying a refinement of topological structure, called convergence structure (CS). CS was applied to serve as the mathematical underpinning for specifying the evolution of compound states by providing an analytical foundation for such state evolution. Such a foundation is geometric in character and is derivable from symmetries in the state space by group-theoretic means and then determining CS with respect to which the symmetries are continuous. Student D. Patten investigated CS methods on discrete structures for the purpose of rigorously formulating hybrid dynamical systems involving discrete structure data-types to aid in developing formal specifications for object-oriented \& similar software involving compound data-types.

\section{SUBJECT TERMS}

Abstract datatypes, convergence structure, topological methods, specification logics, hybrid software, object-oriented software.

\begin{tabular}{|c|c|c|c|c|}
\hline \multicolumn{2}{|l|}{ 16. SECURITY CLASSIFICATION OF: } & $\begin{array}{l}\text { 17. LIMITATION OF } \\
\text { ABSTRACT }\end{array}$ & $\begin{array}{l}\text { 18. NUMBER } \\
\text { OF PAGES }\end{array}$ \\
$\begin{array}{c}\text { a. REPORT } \\
\text { U }\end{array}$ & $\begin{array}{c}\text { b. ABSTRACT } \\
\text { U }\end{array}$ & $\begin{array}{c}\text { c. THIS PAGE } \\
\text { U }\end{array}$ & UU & 33 \\
\hline
\end{tabular}

\begin{tabular}{|l|}
$\begin{array}{l}\text { 19a. NAME OF RESPONSIBLE PERSON } \\
\text { PAUL ALSING }\end{array}$ \\
\hline $\begin{array}{l}\text { 19b. TELEPHONE NUMBER (Include area code) } \\
\text { 315-330-4960 }\end{array}$ \\
\hline
\end{tabular}




\section{Contents}

1 SUMMARY 1

2 INTRODUCTION 2

3 METHODS, ASSUMPTIONS AND PROCEDURES 5

3.1 Differential calculus on nonstandard spaces . . . . . . . . . . . . . 5

3.2 Convergence spaces: an overview . . . . . . . . . . . . . . . 6

3.3 Reflexive digraphs as convergence spaces . . . . . . . . . . . . . 8

3.4 Function spaces . . . . . . . . . . . . . . . . . . . . 9

3.5 (Appendix to section 3: Reflective and coreflective subcategories) . . . . . . . 9

4 RESULTS AND DISCUSSION

4.1 Translation groups and homogeneous convergence spaces . . . . . . . . . . . . 10

4.2 Differential Calculi . . . . . . . . . . . . . . . . . . 11

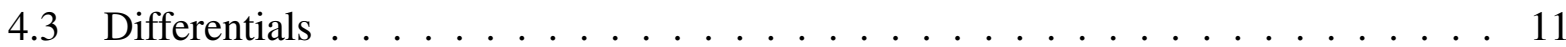

4.4 The Chain Rule . . . . . . . . . . . . . . . . . . . . . . . . 12

4.5 Examples of Differential Calculi . . . . . . . . . . . . . . . . . 13

4.6 Differential Calculi With Nonhomogeneous Objects . . . . . . . . . . . . . . . . 20

4.7 The Chain Rule for Generalized Differential Calculii . . . . . . . . . . . . . . . 21

4.8 Heterotic Dynamical Systems: Classical and Quantum . . . . . . . . . . . . 22

5 CONCLUSIONS $\quad 24$

6 REFERENCES $\quad 25$

7 LIST OF SYMBOLS, ABBREVIATIONS AND ACRONYMS 28 


\section{List of Figures}

1 Unary Boolean operators and their Reed-Akers-Lee-Vichniac hypercube differentials.

The Reed-Akers-Lee-Vichniac linear differentials of unary Boolean operators and the homogeneous differentials of unary Boolean operators are the same as their Reed-Akers-Lee-Vichniac hypercube differentials. . . . . . . . . . . . . . . 15

2 Boolean connectives and their hypercube Reed-Akers-Lee-Vichniac differentials. . 16

3 Boolean connectives and their linear Reed-Akers-Lee-Vichniac differentials. . . . . 18

4 Boolean connectives and their homogeneous differentials. . . . . . . . . . . . . . 19 


\section{SUMMARY}

The research objective, to develop new objective tools to aid in software verification and validation based on topological methods, found that the application of topology to software with composite non-numerical, non-metric data-types, proved to be too course-grained to provide a mathematical foundation for rigorously reasoning about the evolution of the composite state of executing software but overcame this difficulty by applying a refinement of topological structure, called convergence structure $(\mathrm{CS})$.

CS was applied to serve as the mathematical underpinning for specifying the evolution of composite states by providing an analytical foundation for the evolution of such states. Such a foundation is geometric in character and is derivable from symmetries in the state space by group-theoretic means and then determining optimal CS with respect to which the symmetries are continuous. This approach is justified because the evolving values of variables that take entire data-structures as their values cannot in general be numerically encoded without either having to subjectively assess the appropriateness of a particular encoding, or else encounter questionbegging circularities. At the logical level in an actual computing device, composite datatype values are networks strewn through the device's memory; think of a variable whose type is an array of balanced trees. Some means, structural in nature, is required to rigorously specify the evolution of composite states involving non-numerical, non-metric components. Composite datatypes cannot in general be numerically encoded without either having to subjectively assess the appropriateness of a particular encoding, or else encounter question-begging circularities. Since the fundamental aim of this project has been to objectify correctness assessments of software behavior, the project required, at the very least, a means for specifying correct behavior of the evolution of a composite state having non-numerical components.

The verdict as to whether the application of persistent homology to the problem of obtaining objective signatures that would indicate the present or absence of critical behaviors of executing software remains undecided at the end of the project period, while unsupported work on this problem continues. During the project, in cooperation with Dr. W. A. Miller of Florida Atlantic University (FAU), Dr. Matthew Corne, (NRC Fellow at AFRL/RI), doctoral student Shannon Ray (FAU), and others, a computational experiment was conducted consisting of the application of persistent homology to a 2-dimensional rotational solid that collapses and to 3-dimensional dumbbells that manifest neckpinch singularities. The appearance of critical geometric phenomena in these models was compared with the results produced by persistent homology methods and we concluded via various resolutions that persistent homology does indicate geometric criticality. However, these conclusions were reached by ad hoc means and an objective methodology to reliably determine even the general kinds of dynamical geometric phenomena indicated by persistent homology remains to be developed. Moreover, the geometric phenomena associated with neckpinch singularities occurring in a collapsing dumbbell is vastly simpler than the flow through a state space produced by executing software. Moreover in the course of the project an additional computational experiment was performed with the assistance of consisting of applying persistent homology to detect changes to the structure of a small but nontrivial Petri net for the individual steps of any given trajectory could have been calculated by hand, but for which the persistent homology calculations required a computer. This experiment was carried out with the assistance of Dr. Konstantin Mischaikow (Rutgers University), Dr. Vidit Nanda (then of Rutgers Univer-sity), Mr. Anthony Ford (then of AFRL/RI) and Dr. Jonathan McDonald (then an NRC Fellow

Approved for Public Release; Distribution Unlimited. 
at AFRL/RI). The ad hoc result was that gross changes in the dynamics of the Petri net under various loads could be detected, but more subtle changes in the dynamics could not. This does not deprecate persistent homology as an approach; rather it indicates that a vast amount of close investigation of the relationship between the Betti spectra as a function of a filtration parameter produced by persistent homology, and the dynamically evolving underlying geometry is needed, and will best be served by understanding much more about the dynamics of such flows, first.

Accordingly, the project focused on developing the mathematically rigorous specification and verification of dynamical systems involving variables with compound type. We call these dynamical systems heterotic dynamical systems. It is easy to see a program with variables of composite type as a heterotic dynamical system, and it is to be expected, therefore, that there will be components of the evolving computation states associated with the execution of these programs whose type is essentially a class of discrete structures such as linked lists for example.

The capability to rigorously define heterotic dynamical systems and consequently a specification logic for them is due to key properties of the collection of all convergence spaces. Convergence spaces and the continuous functions among them form a large Cartesian-closed Category, which is simultaneously a Cartesian closed completion of the category of topological spaces and the category of reflexive directed graphs whose arrows are directed graph homomorphisms. Within this category the conditions for the functions to among the convergence spaces are the same: preservation of converging filters. Thus, homorphisms and continuous functions are revealed to be the same kind of mathematical object: a function that preserves convergence of filters.

A doctoral student, D. R. Patten, investigated CS methods on discrete structures for the purpose of rigorously formulating heterotic dynamical systems involving discrete structure data-types. Patten's investigation will aid in developing formal specifications for object-oriented and similar software involving composite data-types. Patten successfully defended his doctoral dissertation embodying this research in June, 2014. Patten's work identified a substantial amount of practical detail to exploit when constructing differential calculus among spaces with components that are discrete structures such as networks.

\section{INTRODUCTION}

The application of topology to verifying and validating software with composite non-numerical, non-metric data-types, is too course-grained to provide a mathematical foundation for rigorously reasoning about the evolution of the composite state of executing software. A topology on a set of elements is about nearness: which elements are near to which elements. The individual elements can have a complicated internal structure, and there may not be enough separation strength [20] to numerically characterize the nearness notion with a metric or with continuous maps into the real numbers [42]. Accordingly, applications of computational algebraic topology that face such limitations require appropriate adaptations of topological methods. The objective of this research therefore became that of adapting techniques of computational algebraic topology to much finergrained topology-like convergence structures of which topologies are a special case [10].

We know that such an adaptation can be carried out for the following reason: A fundamental motive for topology is to capture the structural essentials of the notion of continuity shorn of incidental features, such as quantitative characterizations of continuity familiar from the $\epsilon, \delta$ definitions of elementary calculus. Nevertheless, the relatively coarse-grained nature of topologies seems to be unsuitable for the analogous characterization of differentiation, while convergence structures do allow for a structural non-quantitative characterization of differentiation seamlessly with classical

Approved for Public Release; Distribution Unlimited. 
analysis [14]. There are several reasons underlying this capability of convergence spaces; i.e. a convergence space is a nonempty set equipped with a convergence structure.

1. The fine-grained character of convergence structures: The distinction between convergence structure and topological structure can be succinctly grasped by considering the specialization of both to directed graphs with self-loops, i,e, with a reflexive edge relation. A directed graph with a reflexive, and transitive edge relation is a topological space where every vertex occurs in a smallest open set containing that vertex and consisting of all adjacent vertices. A directed graph with a merely reflexive edge relation is a convergence space. Thus by coarsening a directed graph by transitively closing its edge relation one moves from a convergence structure to the induced topological structure.

2. The Cartesian-closure of the category of convergence spaces: In a Cartesian closed category [33], among other features, each collection of $\operatorname{arrows} \operatorname{Hom}(\mathrm{A}, \mathrm{B})$ from an object $A$ to an object $B$ is represented in the category by at least one object. The collection of all convergence spaces forms a Cartesian-closed category CONV in which the objects are convergence spaces and the arrows are continuous functions among them [24] [17]. As objects of the category, the function spaces $Y^{X}$ of continuous functions from $X$ to $Y$ are themselves convergence spaces with a convergence structure that is determined by the convergence structures on $X$ and $Y$ in a uniform manner throughout CONV. CONV is a cartesian-closed extension of both the category RDIGRAPH, the category of directed graphs with self-loops where directed graph homomorphisms are the arrows, as well as the category TOP of topological spaces with continuous functions as the arrows. RDIGRAPH and TOP are full subcategories of $\mathrm{CONV}$, i.e. no arrows are lost in restricting to these subcategories.

3. A differential calculus on a collection of spaces: In prior work the PI formulated differential calculus on convergence spaces. [14]. A differential calculus is determined by the relationship that function $g$ is a differential of function $f$ at a point $x$, together with a functorial chain rule. In other words, the (covariant) derivative operator maps points in the domain of $f$ to differentials. The functoriality of the chain rule is what yields calculus; for example the other familiar rules of elementary calculus on Euclidean spades, such as the product rule follow from the chain rule together with differentials of sum, product and projection, and the rules for cartesian products of functions. To obtain a differential calculus using non-quantitative, non-metric structural means on collection of spaces, a functorial chain rule must be obtained, and functions among convergence spaces must be selected to serve as differentials. For a calculus to be usefully robust, we prefer, but do not require, that the projection functions and diagonal functions such as

$$
(x, y) \mapsto x \quad \text { and } \quad x \mapsto(x, x)
$$

have suitable differentials. The continuity of these sorts of functions having to do with Cartesian products, as well as continuity of the composition operator that allows to combine two functions in sequence, are the features of the Cartesian-closedness of CONV essential to our purpose.

4. The focus on computational algebraic topology: To date this is largely based on homology for reasons of computational tractability. The simplex is the basic mathematical object at the 
core of mainstream homology. The standard geometrical representation of an $n$-dimensional simplex is the convex hull of $n+1$ orthonormal basis vectors in $n+1$ dimensional Euclidean space. However, in a more general topological space $X$, even a metric space, a simplex is a continuous mapping from a standard geometrical representation simplex into $X$. The target space of such a continuous map can just as well be a convergence space, i.e. a nonempty set equipped with a convergence structure, and must be, if the target space is a dynamical system's state space with components of the states that consist of discrete data-structures. Since RDIGRAPH occurs in multiple guises as a full subcategory of CONV in a special way: there are continuous functions among reflexive digraphs; i.e. there are continuous functions among discrete structures. This shows that homomorphisms, are a special case of continuous functions. This observation does not hold within the category of topological spaces, and this is where CONV shines: The set $Y^{Y}$ of all continuous functions on $Y$ is also a convergence space, where if $Y$ is a reflexive digraph, then $Y^{Y}$ is the set of all endomorphisms on $Y$. $Y^{Y}$ has subspaces consisting of abelian groups of automorphisms of $Y$. These subspaces have group endomorphisms on them from which arise ring modules that are semi-regular actions on $Y$, decomposing $Y$ into orbits of the action. The endomorphisms in the module are the translations of $Y$ in exactly the same sense as affine functions on Euclidean spaces are translations of those spaces. Since these translations are also automorphsisms of $Y$ they are symmetries of $Y$. These ring modules are, in effect, the natural geometries inherent in the convergence structure on $Y$. Whenever the ring modules are algebraic fields, the individual orbits into which $Y$ decomposes are vector spaces. Thus $Y$ is revealed as a disjoint union of vector spaces in such cases, but is always a disjoint union of ring modules in any case. Ring modules may be only nearly vector spaces but for that fact that not all of the nonzero scalars need to be invertible and arise naturally in functional analysis as, for example, the space of differential forms on a smooth manifold. Vector spaces are special cases of ring modules. The work in this project culminated in the study of the persistent homology to a 2-dimensional rotational solid that collapses and to 3-dimensional dumbbells that manifest neckpinch singularities. This study indicated that applications of persistent homology to high dimensional complicated dynamical systems is premature [5, 15].

The project focused on developing the mathematically rigorous specification and verification of dynamical systems involving variables with compound type. We call these dynamical systems heterotic dynamical systems. There is a very large degree of freedom in the choice of convergence structure with which to equip a state space. This range of choice must be severely narrowed if pre-existing software is to be modeled by heterotic dynamical systems in an objective sense. Convergence structure, like topological structure but more sensitively, determines in a structural sense, what elements that are near to each other. Specific executions of software produce trajectories in their computation space that can be regarded as continuous images (remember that with convergence spaces our naive intuitions about what continuity is often fail, and this is true even with conventional applications of topology) of time, either modeled as the integers in the case of fully autonomous digital computation, or as the real numbers if the software, for example, is part of a control system with feedback. The execution trajectories determine a finest (and, therefore, contravariantly a smallest) convergence structure on the state space. The point is that the execution trajectories uniquely determine the CS, and hence the geometric structure on the state space, and conversely, for a heterotic dynamical system defined relative to an absolute time, the convergence

Approved for Public Release; Distribution Unlimited. 
structure on the state space constrains the possible trajectories.

A doctoral student, D. R. Patten, [36] investigated CS methods on discrete structures for the purpose of rigorously formulating heterotic dynamical systems involving discrete structure datatypes. Patten's investigation will aid in developing formal specifications for object-oriented and similar software involving composite data-types. Patten successfully defended his doctoral dissertation embodying this research in June, 2014. Patten's work identified a substantial amount of practical detail to exploit when constructing differential calculus among spaces with components that are discrete structures such as networks.

\section{METHODS, ASSUMPTIONS AND PROCEDURES}

\subsection{Differential calculus on nonstandard spaces}

With topology, continuity of functions generalizes from the context of classical analysis to a huge collection of structures, the topological spaces. ity. Differentiability also generalizes to all topological spaces, and beyond (as does continuity) to all convergence spaces. Just as continuity neither presupposes any seperation strength nor any notions of linearity, neither does differentiability. The familiar differential calculus on Euclidean spaces is of course intrinsically dependent on the vector space structure, but this is due to the choice of functions used to serve as differentials, and the consequent determination of the conditions under which functions are differentiable. What counts is the relation "differential $g$ is a differential of $f$ at $x$ ". Unless we demand of $g$ that it satisfy some kind of linearity property, linearity does not intrinsically enter into the relation. CONV, the Cartesian-closed category of convergence spaces and continuous maps [30], is central in this generalization. The spaces and functions of interest are naturally organized into categories and it is important to note the nature of the containments and embeddings that are involved. For example, any method for constructing a differential calculus for mappings between arbitrary convergence spaces gives such a method for all reflexive digraphs and all topological spaces.

The category CONV includes all topological spaces but enjoys several substantial advantages over the category TOP of topological spaces. Importantly for computation, CONV contains all reflexive directed graphs (finite and infinite). Among digraphs, continuity is the property of being edge-preserving, i.e. a digraph homomorphism. But, powerfully, and unlike TOP, CONV is a Cartesian-closed category. Several immediate consequences of Cartesian-closure and the relationship between TOP and CONV are: (1) convergence spaces preserve the notion of continuity on topological spaces; (2) convergence spaces allow fine control over continuity, and in various circumstances permit us to strengthen the conditions for continuity; (3) there is a uniform way of regarding all spaces of continuous functions as convergence spaces (enabling hybrid functional analysis) and what is key to differential calculi: (4) the composition operation on finite products of function spaces is continuous, as is the evaluation function.

In the classical differential calculus, differentials of a small class of basic functions are obtained $a b$ initio. Differentials of functions generated from the basic functions by generalized composition are obtained via the chain rule. Differentiation factors out of composition, and the identity function is its own differential everywhere. It is often underappreciated by students of calculus that familiar rules follow from the chain rule and details of the differentials of various basic functions. For example, the product rule follows from the chain rule and the differential of the multiplication operation. Also, proofs of the chain rule in elementary calculus texts often adopt unnecessary hypotheses of continuous differentiability. These proofs do, correctly, use the continuity of

Approved for Public Release; Distribution Unlimited. 
multiplication, but this actually is a projection of the continuity of the composition operation on differentials in CONV.

Category-theoretic characterizations of heterotic models of computation were introduced by Stepney et.al. in [40]. Heterotic models of computation combine computational models such as classical/quantum, digital/analog, synchronous/asynchronous, imperative/functional/relational, etc. to obtain increased computational power, both practically and theoretically. In the project we had been developing heterotic dynamical systems consistent with Stepney et.al.'s notions, but had been unaware of their work and terminology until after their work appeared. These systems involve the evolution of closely coupled variables of broadly varying type, some discrete-valued, some continuous-valued, and some with values that are not quantitative. The definition, representation and manipulation of differentials is non-obvious in such circumstances.

There is another important observation that is obscured in the elementary calculus of realvalued functions on the real numbers (elementary calculus in one dimension): derivatives of functions that are differentiable on, say, an interval, are functions that are differential valued. (This is already observable in multi-dimensional elementary calculus.) The function space of differentials arising in this context is the space of linear functions from the reals to the reals as a subspace of the convergence space of continuous functions from the reals to the reals in CONV. This space of differentials is homeomorphic to the space of real numbers equipped with the standard, Euclidean, topology. Using the homeomorphism, one then identifies the differentials with real numbers and thereby obtains real-valued derivatives. Much of this is invisible in standard calculus texts, even at fairly advanced levels. It is important to note that in more general contexts the homeomorphisms through which such identifications are made will not always exist. In particular, notions of continuous differentiability then require that derivatives be differential-valued and require that something like topological structure (in our case, convergence structure) on the function spaces that arise be taken into consideration.

If it is insisted upon that differentials be linear functions, then several options present themselves. Most stringently, continuous ring-module structures can be extracted from the symmetries in the convergence structures on the spaces involved. Less stringently, functions in the role of linear functions are determined by the continuous homormorphisms among the translation groups (cf. section on the spaces involved. When the translation groups are both full and Abelian we obtain continuous ring modules. However, blind insistence upon linearity is misguided. Even in Euclidean spaces, one obtains an interesting differential calculus by taking the continuous homogeneous functions of order 1 (i.e. $f(a x)=a f(x))$ as the differentials. One even obtains a more general but less interesting calculus by dropping the continuity requirement on the differentials, but preserving continuity of composition.

Over time, a number of researchers have sought to generalize differentiability to spaces where the generalization is non-obvious. Some of the more serious and sophisticated results in this direction have employed one or another restriction of the notion of convergence space, often near to pre-topological spaces, or else stayed within TOP $[7,8,11,12,22,23,28,31,34,35]$. These explorations assumed the existence of additional structure characterizing linearity. [31] recognized the importance of Cartesian-closure for obtaining a robust chain-rule.

\subsection{Convergence spaces: an overview}

A filter on a set $X$ is a collection of subsets of $X$ closed under finite intersection and reverse inclusion. A proper filter is a filter which does not contain the empty set as a member. Let $\Phi(X)$ 
denote the set of all filters on $X$. For a subset $A$ of $X,\{B \mid A \subseteq B \subseteq X\}$ is a member of $\Phi(X)$. We denote this filter by $[A]$. In the special case where $A$ is a singleton $\{x\}$ we denote $[A]$ by $[x]$ and call this the point filter at $x$.

Definition 3.1. A convergence structure on $X$ is a relation $\downarrow$ (read as "converges to") between members of $\Phi(X)$ and members of $X$ such that for each $x \in X:$ (1) $[x]$ converges to $x$, and (2) the set of filters converging to $x$ is closed under reverse inclusion. A pair $(X, \downarrow)$ consisting of a set $X$ and a convergence structure $\downarrow$ on $X$ is called a convergence space.

A function $f: X \longrightarrow Y$ where $X$ and $Y$ are sets, induces functions $\hat{f}: 2^{X} \longrightarrow 2^{Y}$ and $\hat{\hat{f}}: \Phi(X) \longrightarrow \Phi(Y)$. $\hat{f}$ is defined by $\hat{f}(A)=\{f(a) \mid a \in A\}$, which we call the $f$-image of $A$ w.r.t $f$. For $\mathcal{F} \in \Phi(X)$ note that the collection of all supersets of $f$-images of members of $\mathcal{F}$ forms a filter which we call $\hat{f}(F)$. Hereafter we overload notation and drop the ${ }^{\wedge}$ and $\hat{\wedge}$ annotations.

When convenient, we will refer to a convergence space $(X, \downarrow)$ by its carrier, $X$.

Definition 3.2. Let $f: X \longrightarrow Y$ where $X$ and $Y$ are convergence spaces, and let $x_{0} \in X$. $f$ is continuous at $x_{0}$ iff for each $\mathcal{F} \in \Phi(X)$, if $\mathcal{F} \downarrow x_{0}$ in $X$, then $f(F) \downarrow f\left(x_{0}\right)$ in $Y$. $f$ is continuous iff $f$ is continuous at every point of $X$.

Continuity can be characterized in terms of filter members, which play a role analogous to the role played by neighborhoods, as supersets of open sets, in topological spaces.

Proposition 3.1. Let $f: X \longrightarrow Y$ where $X$ and $Y$ are convergence spaces, and let $x_{0}$ be a point of $X . f$ is continuous at $x_{0}$ iff for every filter $\mathcal{F}$ converging to $x_{0}$ in $X$, there is a filter $\mathcal{G}$ converging to $f\left(x_{0}\right)$ in $Y$ such that $(\forall V \in \mathcal{G})(\exists U \in \mathcal{F})[f(U) \subseteq V]$.

Definition 3.3. A homeomorphism between two convergence spaces is a continuous bijection whose inverse is continuous.

Definition 3.4. Let $x$ be a point of a convergence space $X$, and let $U$ be a subset of $X$. $U$ is said to be a neighborhood of $x$ iff $U$ belongs to every filter converging to $x$.

Definition 3.5. [19] A convergence space $(X, \downarrow)$ is said to be a pretopological space if and only if $\downarrow$ is a pretopology, i.e. for each $x \in X$, the collection of all neighborhoods of $x$ converges to $x$.

Proposition 3.2. Let $f: X \longrightarrow Y$ where $X$ and $Y$ are pretopological spaces, and let $x_{0} \in X$. $f$ is continuous at $x_{0}$ iff for every neighborhood $V$ of $f\left(x_{0}\right)$, there is a neighborhood $U$ of $x_{0}$ such that $f(U) \subseteq V$.

It is evident that every topological space is a pretopological space (cf. [19, 18, 29]), and that the convergence space notion of continuity and the topological space notion of continuity coincide for topological spaces. As indicated in the introduction, the spaces and functions of interest to us are naturally organized as categories. The main categories of interest in this paper are:

CONV the category of convergence spaces and continuous functions

PreTOP the category of pretopological spaces and continuous functions

TOP the category of topological spaces and continuous functions

RDIGRAPH the category of reflexive digraphs (i.e. directed graphs

PostDISCRETE

with a loop at each vertex) and edge-preserving functions

the full subcategory of CONV whose objects are the

post discrete convergence spaces

Approved for Public Release; Distribution Unlimited. 
TOP is a full subcategory of PreTOP, which, in turn, is a full subcategory of CONV. Both of these full inclusions are reflective, via induced pretopology and induced topology operations, respectively. RDIGRAPH is isomorphic to PostDISCRETE, which, in turn, embeds into PreTOP. ${ }^{1}$

The reflection functor PreT from CONV to PreTOP can be realized by letting the carrier of $\operatorname{PreT}(X)$ be the carrier of $X$, and and letting a filter $\mathcal{F}$ converge to a point $x$ in $\operatorname{PreT}(X)$ iff the collection of all neighborhoods of $x$ in $X$ is a subcollection of $\mathcal{F}$.

Similarly, the reflection functor T from PreTOP to TOP can be realized by letting the carrier of $\mathrm{T}(X)$ be the carrier of $X$, and defining the topology on $\mathrm{T}(X)$ as $\{U \subseteq X \mid U$ is a neighborhood in $X$ of each point of $U\}$

\subsection{Reflexive digraphs as convergence spaces}

RDIGRAPH can be embedded, in more than one way, as a full subcategory of CONV.

Definition 3.6. A convergence space $X$ will be said to be postdiscrete if and only if every convergent proper filter is a point filter.

Proposition 3.3. The postdiscrete pretopological spaces are precisely the discrete topological spaces.

Definition 3.7. Let $(V, E)$ be a reflexive digraph. Induce a convergence structure on $V$ by letting a proper filter $\mathcal{F}$ converge to a vertex $x$ iff $\mathcal{F}=[y]$ for some vertex $y$ with an edge in $E$ from $x$ to $y$.

It is readily verified that if $\left(V_{1}, E_{1}\right)$ and $\left(V_{2}, E_{2}\right)$ are reflexive digraphs, then a function $f: V_{1} \longrightarrow$ $V_{2}$ is continuous (with respect to the induced convergence structures on $V_{1}$ and $V_{2}$ ) iff, for all edges $(x, y)$ in $E_{1}$, the edge $(f(x), f(y))$ is present in $E_{2}$.

Proposition 3.4. The construction in Definition 3.7 embeds $\boldsymbol{R D I G R A P H}$ as a full subcategory of $C O N V$, namely the full subcategory whose objects are the postdiscrete spaces.

This embedding is coreflective. The coreflection functor RDIGRAPH : CONV $\longrightarrow$ RDIGRAPH can be obtained letting the vertices of RDIGRAPH $(X)$ be the members of $X$, and letting an ordered pair $(x, y)$ be an edge of $\operatorname{RDIGRAPH}(X)$ iff $[y] \downarrow x$ in $X$.

Alternatively, RDIGRAPH can be embedded as a full subcategory of PreTOP, and thence as a full subcategory of CONV [41,39], by letting a filter $\mathcal{F}$ converge to a vertex $x$ iff $\{y \mid(x, y) \in E\}$ is a member of $\mathcal{F}$.

In general, this embedding of RDIGRAPH into PreTOP imposes a weaker convergence structure on reflexive digraphs than the embedding in Definition 3.7.

Proposition 3.5. The embedding of RDIGRAPH into PreTOP [41, 39] is the composite of the embedding in Definition 3.7 of RDIGRAPH into CONV with the reflection functor from CONV to PreTOP, and embeds RDIGRAPH as a full, coreflective subcategory of PreTOP, and thence as a full, coreflective subcategory of CONV.

The coreflection functor from PreTOP to RDIGRAPH (and the coreflection functor from CONV to RDIGRAPH via PreTOP) can be obtained in precisely the same way as in Proposition 3.4.

\footnotetext{
${ }^{1}$ The embedding is the restriction of the induced pretopology reflection from CONV to PostDISCRETE
} 
The reflexive digraphs whose induced pretopologies are topological are precisely those in which the underlying binary relation is transitive as well as reflexive. [41, 39]

\subsection{Function spaces}

Unlike TOP and PreTOP, CONV is a Cartesian closed category ([33, 27]):

Definition 3.8. [27] Let $X$ and $Y$ be convergence spaces.

The function space $Y^{X}$ is the set of all continuous functions from $X$ to $Y$, equipped with the convergence structure $\downarrow$ defined as follows:

For each $\mathcal{H} \in \Phi\left(Y^{X}\right)$ and each $f_{0} \in Y^{X}$, let $\mathcal{H} \downarrow f_{0}$ if and only if

for each $x_{0} \in X$ and each $\mathcal{F} \downarrow x_{0},\{\{f(x) \mid f \in H, x \in F\} \mid H \in \mathcal{H}, F \in \mathcal{F}\}$ is a base for a filter which converges to $f\left(x_{0}\right)$ in $Y$.

Definition 3.8 is essentially the same as the definition of function graphs in [26]. This should not be very surprising: RDIGRAPH is a Cartesian closed category (cf. [3]).

Proposition 3.6. [11, 13] Let $X$ and $Y$ be convergence spaces. Then the evaluation function from $Y^{X} \times X$ to $Y$ is continuous.

The following is a very useful consequence of Proposition 3.6.

Corollary 3.1. Let $X, Y$, and $Z$ be convergence spaces. Then the composition function from $Z^{Y} \times Y^{X}$ to $Z^{X}$ is continuous.

\section{5 (Appendix to section 3: Reflective and coreflective subcategories)}

The content of this section, including the second example, is largely taken from [33]. The first example is taken from [25]. Also see [6] and [3].

Throughout this section let $\mathcal{A}$ be a subcategory of a category $\mathcal{B}$.

$\mathcal{A}$ is said to be a reflective subcategory of $\mathcal{B}$ iff there is a reflection functor from $\mathcal{B}$ to $\mathcal{A}$, i.e. a functor $F: \mathcal{B} \longrightarrow \mathcal{A}$ such that each object $B$ of $\mathcal{B}$ is equipped with a reflector, i.e. an arrow $\tau_{B}: B \longrightarrow F B$ in $\mathcal{B}$ such that, for each object $A$ of $\mathcal{A}$ and each arrow $f: B \longrightarrow A$ in $\mathcal{B}$, there is a unique arrow $\hat{f}: F B \longrightarrow A$ in $\mathcal{A}$ such that

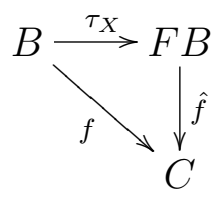

commutes.

If $\mathcal{A}$ is a full, reflective subcategory of $\mathcal{B}$ with reflection functor $F$, then, for each object $A$ of $\mathcal{A}$, $A$ and $F A$ are isomorphic.

For instance, the category of compact Hausdorff spaces and continuous functions is a full, reflective subcategory of Top. The reflection functor $\beta$ is the well-known Stone-Čech functor. The reflector 
from an arbitrary topological space $X$ to $\beta X$ is an embedding if and only if $X$ is completely regular and Hausdorff.

Dually, $\mathcal{A}$ is said to be a coreflective subcategory of $\mathcal{B}$ iff there is a coreflection functor from $\mathcal{B}$ to $\mathcal{A}$, i.e. a functor $F: \mathcal{B} \longrightarrow \mathcal{A}$ such that each object $B$ of $\mathcal{B}$ is equipped with a coreflector, i.e. an arrow $\iota_{B}: F B \longrightarrow B$ in $\mathcal{B}$ such that, for each object $A$ of $\mathcal{A}$ and each arrow $f: A \longrightarrow B$ in $\mathcal{B}$, there is a unique arrow $\hat{f}: A \longrightarrow F B$ in $\mathcal{A}$ such that

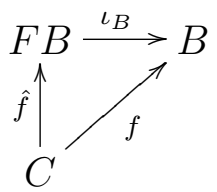

commutes.

If $\mathcal{A}$ is a full, coreflective subcategory of $\mathcal{B}$ with coreflection functor $F$, then, for each object $A$ of $\mathcal{A}, A$ and $F A$ are isomorphic.

For instance, the category of Abelian torsion groups and homomorphisms is a full, coreflective subcategory of the category of Abelian groups and homomorphisms.

\section{RESULTS AND DISCUSSION}

\subsection{Translation groups and homogeneous convergence spaces}

Definition 4.1. An automorphism of a convergence space $X$ is a homeomorphism $f: X \longrightarrow X$.

Definition 4.2. A translation group on a convergence space $X$ is a group $T$ of automorphisms of $X$ such that, for each pair of points $p$ and $q$ of $X$, there is at most one member of $T$ which maps $p$ to $q$. In general, we will denote this unique member of $T$ (if it exists) by $(q-p)$.

Notation: The group operation of a translation group $T$ on a convergence space $X$ will be written additively, whether or not $T$ is Abelian. Furthermore, for all $\tau \in T$ and all $x \in X$, we will write $\tau(x)$ as $x+\tau$

In this notation, the requirement that the translation $(q-p)$ (if it exists) maps $p$ to $q$ becomes the familiar requirement that if $(q-p)$ exists, then $p+(q-p)=q$

A full translation group on a convergence space $X$ is a translation group on $X$ which contains a translation $(q-p)$ for each pair of points $p$ and $q$.

\section{Proposition 4.1.}

i. Every convergence space $X$ can be embedded as a subspace of a convergence space $H X$ which has a full translation group.

ii. $X$ and $H X$ have the same cardinality if and only if the cardinality of $X$ is either zero or infinite.

iii. The embedding of $X$ into $H X$ is onto $H X$ if and only if $X$ is empty. 
iv. If $X$ and $Y$ are arbitrary convergence spaces then every continuous function $f: X \longrightarrow Y$ can be be extended to a continuous function $H f: H X \longrightarrow H Y$.

v. If $f$ is a homeomorphism, then so is $H f$.

An immediate consequence of (ii) in Proposition 4.1 is that, if $X$ is a finite space with (or without) a full translation group, then $H X$ cannot be homomeomorphic to $X$. It should also be noted that, given a particular $f$, the continuous extention $H f$ need not be unique.

Definition 4.3. A convergence space $X$ is homogeneous iff for each pair of points $x_{1}$ and $x_{2}$ of $X$, there is an automorphism of $X$ which maps $x_{1}$ to $x_{2}$

Observation 4.1. A convergence space which has a full translation group must be homogeneous. Furthermore, a full translation group on a nonempty convergence space $X$ must have the same cardinality as $X$.

\subsection{Differential Calculi}

Definition 1. A differential calculus is a category $\mathcal{D}$ in which

i. every object of $\mathcal{D}$ is a triple $\mathcal{X}=(X, 0, T)$ such that $X$ is a convergence space, 0 is a point of $X$ (called the origin of $\mathcal{X}$ ), and $T$ is a full translation group on $X$.

ii. every arrow in $\mathcal{D}$ from an object $\left(X, 0_{X}, T_{X}\right)$ to an object $\left(Y, 0_{Y}, T_{Y}\right)$ is a continuous function from $X$ to $Y$ which maps $0_{X}$ to $0_{Y}$

iii. composition of arrows in $\mathcal{D}$ is function composition.

iv. for every object $\mathcal{X}=\left(X, 0_{X}, T_{X}\right)$, the identity function on $X$ is an arrow in $\mathcal{D}$ from $\mathcal{X}$ to $\mathcal{X}$

v. for each pair of objects $\mathcal{X}=\left(X, 0_{X}, T_{X}\right)$ and $\mathcal{Y}=\left(Y, 0_{Y}, T_{Y}\right)$, the constant function mapping every point of $X$ to $0_{Y}$ is an arrow in $\mathcal{D}$ from $\mathcal{X}$ to $\mathcal{Y}$

In view of Proposition 4.1, the requirement that each object have a full translation group is not unduly restrictive.

\subsection{Differentials}

Let $a \in A \subseteq X$ and let $B \subseteq Y$, where $\mathcal{X}=\left(X, 0_{X}, T_{X}\right)$ and $\mathcal{Y}=\left(Y, 0_{Y}, T_{Y}\right)$ are objects of a differential calculus $\mathcal{D}$. Let $f: A \longrightarrow B$ be an arbitrary function.

Let $L \in \mathcal{D}(\mathcal{X}, \mathcal{Y})$, where $\mathcal{D}(\mathcal{X}, \mathcal{Y})$ is the set of all arrows in $\mathcal{D}$ from $\mathcal{X}$ to $\mathcal{Y}$, equipped with the subspace convergence structure inherited from the function space $Y^{X}$ in CONV.

Definition 2. $L$ is a differential of $f$ at $a$ iff

for every $\mathcal{F} \downarrow a$ in $A$, there is some $\mathcal{H} \downarrow L$ in $\mathcal{D}(\mathcal{X}, \mathcal{Y})$ such that

i. $\mathcal{H} \subseteq[L]$, and 
ii. for every $H \in \mathcal{H}$, there is some $F \in \mathcal{F}$ such that for every point $x \in F$, there is at least one function $t \in H$ such that

$$
t\left(x+\left(0_{X}-a\right)\right)=f(x)+\left(0_{Y}-f(a)\right)
$$

(In Definition 2, $\left(f(a)-0_{Y}\right) \circ t \circ\left(0_{X}-a\right)$ is an extrapolant of $f$ through $(a, f(a))$ and $(x, f(x))$.)

Definition 3. A function from $A$ to $B$ is differentiable (respectively, uniquely differentiable) at a point $a$ iff it has at least one (respectively, precisely one) differential at $a$.

A function from $A$ to $B$ is differentiable (respectively, uniquely differentiable) iff it is differentiable (respectively, uniquely differentiable) at each point of $A$.

\subsection{The Chain Rule}

As we indicated in the introduction, the chain rule is among the central roles in our differential calculi. In elementary calculus for example, the product rule follows from the chain rule after obtaining the differential of the multiplication operation.

Example 4.1. Expressed in terms of differentials, the product rule for real-valued functions of a real variable reduces to matrix multiplication (i.e. composition of linear functions).

$$
\begin{aligned}
D_{x}(\text { mult } \circ(f, g)) & =D_{(f, g)(x)} \operatorname{mult} \circ D_{x}(f, g) \\
& =D_{(f(x), g(x))} \operatorname{mult} \circ\left(D_{x} f, D_{x} g\right) \\
& =[g(x) f(x)]\left[\begin{array}{c}
D_{x} f \\
D_{x} g
\end{array}\right] \\
& =g(x) D_{x} f+f(x) D_{x} g
\end{aligned}
$$

Returning to our more general setting, let $a \in A \subseteq X$, let $B \subseteq Y$, and let $C \subseteq Z$, where $\mathcal{X}=\left(X, 0_{X}, T_{X}\right), \mathcal{Y}=\left(Y, 0_{Y}, T_{Y}\right)$, and $\mathcal{Z}=\left(Z, 0_{Z}, T_{Z}\right)$ are objects of a differential calculus $\mathcal{D}$. Let $f: A \longrightarrow B$ and $g: B \longrightarrow C$ be arbitrary functions.

Let $K: \mathcal{X} \longrightarrow \mathcal{Y}$ and $L: \mathcal{Y} \longrightarrow \mathcal{Z}$ be arrows of $\mathcal{D}$

Theorem 4.1. (Chain Rule) Suppose that $f$ is continuous at $a$. Also suppose that $K$ is a differential of $f$ at $a$, and $L$ is a differential of $g$ at $f(a)$.

Then $L \circ K$ is a differential of $g \circ f$ at $a$.

proof: Let $\mathcal{F}$ be a filter converging to $a$ in $X$. Since $K$ is a differential of $f$ at $a$, there is some $\mathcal{G} \downarrow K$ in $\mathcal{D}(\mathcal{X}, \mathcal{Y})$ such that $\mathcal{G} \subseteq[K]$ and, for every $G \in \mathcal{G}$, there is some $F_{1, G} \in \mathcal{F}$ such that for each point $x \in F_{1, G}$ there is some function $s_{G, x} \in G$ such that

$$
s_{G, x}\left(x+\left(0_{X}-a\right)\right)=f(x)+\left(0_{Y}-f(a)\right)
$$

On the other hand, since $f$ is continuous at $a$, we have $f(\mathcal{F}) \downarrow f(a)$ in $B$. Since $L$ is a differential of $g$ at $f(a)$, there is some filter $\mathcal{H} \downarrow L$ in $\mathcal{D}(\mathcal{Y}, \mathcal{Z})$ such that $\mathcal{H} \subseteq[L]$ and, for every $H \in \mathcal{H}$, there is some $N_{H} \in f(\mathcal{F})$ such that for each point $y \in N_{H}$, there is some function $t_{H, y} \in V$ such that

$$
\begin{gathered}
t_{H, y}\left(y+\left(0_{Y}-f(a)\right)\right)=g(f(x))+\left(0_{Z}-g(f(a))\right) \\
\text { Approved for Public Release; Distribution Unlimited. }
\end{gathered}
$$


Consider such a set $N_{H}$. By definition, $N_{H} \in f(\mathcal{F})$, i.e. there is some $F_{2, H} \in \mathcal{F}$ such that

$$
f\left(F_{2, H}\right) \subseteq N_{H}
$$

By (2), for each point $x \in F_{2, H}$, we have

$$
t_{H, f(x)}\left(f(x)+\left(0_{Y}-f(a)\right)\right)=g(f(x))+\left(0_{Z}-g(f(a))\right)
$$

Next, note that $\left\{\left\{h_{2} \circ h_{1} \mid h_{1} \in G, h_{2} \in H\right\} \mid G \in \mathcal{G}, H \in \mathcal{H}\right\}$ is a basis for a filter $\mathcal{J}$ on $\mathcal{D}(\mathcal{X}, \mathcal{Z})$, and that $\mathcal{J} \subseteq[L \circ K]$.

By joint continuity of composition (Corollary 3.1), $\mathcal{J} \downarrow L \circ L$ in $\mathcal{D}(\mathcal{X}, \mathcal{Z})$.

Let $J$ be an arbitrary member of $\mathcal{J}$. There exist $G \in \mathcal{G}$ and $H \in \mathcal{H}$ such that

$$
\left\{h_{2} \circ h_{1} \mid h_{1} \in G, h_{2} \in H\right\} \subseteq J
$$

Let $F=F_{1, G} \cap F_{2, H}$. Then $F \in \mathcal{F}$. For each point $x \in F$, we have $s_{G, x} \in G$ and $t_{H, f(x)} \in H$, and therefore $t_{H, f(x)} \circ s_{G, x} \in J$. Furthermore, by (1) and (3),

$$
t_{H, f(x)}\left(s_{G, x}\left(x+\left(0_{X}-a\right)\right)\right)=t_{H, f(x)}\left(f(x)+\left(0_{Y}-f(a)\right)\right)=g(f(x))+\left(0_{Z}-g(f(a))\right)
$$

Thus, $\left(g(f(a))-0_{Z}\right) \circ t_{H, f(x)} \circ s_{G, x} \circ\left(0_{X}-a\right)$ is the required extrapolant of $g \circ f$ through $(a, g(f(a)))$ and $(x, g(f(x)))$.

Since the selection of $x$ is arbitrary (once $G$ and $H$ have been chosen), $L \circ K$ is indeed a differential of $g \circ f$ at a.

\subsection{Examples of Differential Calculi}

In all examples, let $\mathbf{R}$ be the real line, and let $\mathbf{N}$ be the set of all natural numbers.

\section{Example 4.2. The classical Newton-Leibniz differential calculus of real variables}

The objects of this differential calculus are the spaces $\mathbf{R}^{n}(n \in \mathbf{N})$, equipped with their usual (Euclidean) convergence structures, their usual origins, and their usual translation groups.

The arrows of this calculus are the $\mathbf{R}$-linear functions.

In this calculus, differentiability and unique differentiability are equivalent, and a function $f$ has a differential at a point $p$ iff $f$ is differentiable (in the usual sense) at $p$.

\section{Example 4.3. A directional calculus of real variables}

This is an extension of the Newton-Leibniz calculus of real variables. Again, the carriers of objects are the sets $\mathbf{R}^{n}(n \in \mathbf{N}$ ), equipped with their usual origins and their usual translation groups.

However, each $\mathbf{R}^{n}$ will be equipped with two convergence structures, and thus will be be the carrier of two distinct objects.

One of these convergence structures is the familiar Euclidean structure.

The other convergence structure which we impose on $\mathbf{R}^{n}$ is defined as follows:

A filter $\mathcal{F}$ will be said to converge to a point $p$ iff there is some unit vector $q$ such that

$$
\{p+\alpha q|\alpha \in \mathbf{R},| \alpha \mid<\epsilon\} \in \mathcal{F}
$$

for every real number $\epsilon>0$. We call this convergence structure the directional convergence structure on $\mathbf{R}^{n}$. 
For $n=1$, the directional convergence structure coincides with the Euclidean structure. For $n>1$, this directional convergence structure is stronger than the Euclidean structure, and is not pretopological.

The arrows of this calculus are the continuous $\mathbf{R}$-homogeneous functions of degree one.

Again, differentiabilty and unique differentiabilty are equivalent. Observe that a function $f$ from $\mathbf{R}^{m}$ with its directional convergence structure to $\mathbf{R}^{n}$ with its Euclidean convergence structure has a differential at a point $p$ iff $f$ has directional derivatives in all directions at $p$.

\section{Example 4.4. The one-sided calculus of a single function of a real variable}

The only object of this differential calculus is the real line $\mathbf{R}$, equipped with its usual (Euclidean) convergence structure, its usual origin, and its usual translation group.

Define the arrows from $\mathbf{R}$ to itself to be the set of all functions $L: \mathbf{R} \longrightarrow \mathbf{R}$ such that

$$
\begin{aligned}
& \text { for all } x, y \in \mathbf{R} \text { and every } \alpha \geq 0 \\
& \qquad L(\alpha(x+y))=\alpha L(x)+\alpha L(y)
\end{aligned}
$$

In this calculus, a function has a differential at a point $p$ iff it has both a left-sided derivative at $p$ and a right-sided derivative at $p$. As in the previous examples, differentiability and unique differentiability are equivalent.

\section{Example 4.5. The Reed-Akers-Lee-Vichniac calculus of Boolean partial derivatives} $([38,4,32]$.

Let $\mathbf{B}$ be the two-element field, i.e. the field of integers modulo two.

Let $m$ be a natural number, and let $f$ be an arbitrary function from $\mathbf{B}^{m}$ to B. Following [38], [4], and [32], define the $i^{\text {th }}$ partial derivative of $f$ at a point $x$ as the difference quotient

$$
\left.\frac{\partial f}{\partial x_{i}}\right|_{x}=\frac{f\left(x_{1}, x_{2}, \ldots, x_{m}\right)-f\left(x_{1}, x_{2}, \ldots, x_{i-1}, \neg x_{i}, x_{i+1}, x_{i+2}, \ldots, x_{m}\right)}{x_{i}-\neg x_{i}}
$$

Observe that this is always well-defined. Also observe that, since the arithmetic is modulo two, this definition simplifies to

$$
\left.\frac{\partial f}{\partial x_{i}}\right|_{x}=f\left(x_{1}, x_{2}, \ldots, x_{m}\right)+f\left(x_{1}, x_{2}, \ldots, x_{i-1}, \neg x_{i}, x_{i+1}, x_{i+2}, \ldots, x_{m}\right)
$$

In other words, the $i^{\text {th }}$ partial derivative of $f$ at $\left(x_{1}, x_{2}, \ldots x_{m}\right)$ is 1 if changing the value of $x_{i}$ changes the value of $f\left(x_{1}, x_{2}, \ldots, x_{m}\right)$, and is 0 otherwise.

Following [32] and [43], if $m$ and $n$ are natural numbers, define the Jacobean matrix of an arbitrary function $F$ from $\mathbf{B}^{m}$ to $\mathbf{B}^{n}$ as the matrix of partial derivatives of the component functions of $F$. Note that this too is always well-defined. ${ }^{2}$

Higher-order partial derivatives, mixed partials, etc., are defined in [9] in the obvious way.

Many of the rules of the classical differential calculus of real variables, such as the product rule, also hold for Boolean derivatives [9]. However, it is notorious that the conclusion of the chain rule does not always hold, if partial derivatives are involved [43]. We can explain this.

\footnotetext{
${ }^{2}$ In [32], the Jacobean matrix of $F$ is called the differential of $F$.
} 
For each natural number $n$, take the members of $\mathbf{B}^{n}$ as the vertices of a digraph, with an edge from a vector $\vec{a}=\left(a_{1}, a_{2}, \ldots, a_{n}\right)$ to a vector $\vec{b}=\left(b_{1}, b_{2}, \ldots, b_{n}\right)$ iff the Hamming distance between $\vec{a}$ and $\vec{b}$ is at most 1 , i.e. there is at most one $i$ such that $a(i) \neq b(i)$.

In other words, take the members of $\mathbf{B}^{n}$ as the vertices of a hypercube with two-way edges, and with a loop at each vertex. Equip $\mathbf{B}^{n}$ with the postdiscrete convergence structure induced by this digraph (cf. Definition 3.7).

In short, the proper filters which converge to a bit vector $\vec{a}$ are precisely the point filters $[\vec{b}]$ such that the bit vector $\vec{b}$ differs from $\vec{a}$ in at most one bit.

Take these spaces $\mathbf{B}^{n}$ as the carriers of the objects of a differential calculus. For each $n$, take the zero vector of length $n$ as the origin of $\mathbf{B}^{n}$.

The group generated by the flips $h_{1}, h_{2}, \ldots, h_{n}$ is taken as the translation group of $\mathbf{B}^{n}$, where (as one would expect) $h_{k}(\vec{b})$ is obtained from bit vector $\vec{b}$ by changing the $k^{\text {th }}$ bit of $\vec{b}$ (and leaving every other bit unchanged).

For each $m$ and $n$, take the continuous $\mathbf{B}$-linear functions from $\mathbf{B}^{m}$ to $\mathbf{B}^{n}$ as the differentials from $\mathbf{B}^{m}$ to $\mathbf{B}^{n}$.

In particular, there are precisely two differentials between $\mathbf{B}$ and itself, namely, the constant function which returns zero, and the identity function.

In this differential calculus, as in the previous two examples, differentiability and unique differentiability are equivalent.

It is readily verified that if a function $F$ from $\mathbf{B}^{m}$ to $\mathbf{B}^{n}$ has a differential $L$ at a bit vector $\vec{x}$, then $L$ is the $\mathbf{B}$-linear function whose matrix (with respect to the standard bases of $\mathbf{B}^{m}$ and $\mathbf{B}^{n}$ ) is the Jacobean matrix of $F$.

\begin{tabular}{c|c|c} 
Function & Differential at 0 & Differential at 1 \\
\hline constant with value 0 & constant with value 0 & constant with value 0 \\
constant with value 1 & constant with value 0 & constant with value 0 \\
identity & identity & identity \\
$\neg$ & identity & identity \\
\hline
\end{tabular}

Figure 1: Unary Boolean operators and their Reed-Akers-Lee-Vichniac hypercube differentials. The Reed-Akers-Lee-Vichniac linear differentials of unary Boolean operators and the homogeneous differentials of unary Boolean operators are the same as their Reed-Akers-Lee-Vichniac hypercube differentials.

Every function between $\mathbf{B}$ and itself is differentiable everywhere. 


\begin{tabular}{|c|c|c|c|c|}
\hline \multirow[t]{2}{*}{ Function } & \multicolumn{4}{|c|}{ Differential at } \\
\hline & $(0,0)$ & $(0,1)$ & $(1,0)$ & $(1,1)$ \\
\hline const $_{0}$ & const $_{0}$ & const $_{0}$ & const $_{0}$ & const $_{0}$ \\
\hline const $_{1}$ & const $_{0}$ & const $_{0}$ & const $_{0}$ & const $_{0}$ \\
\hline $\operatorname{proj}_{1}$ & $\operatorname{proj}_{1}$ & $\operatorname{proj}_{1}$ & $\operatorname{proj}_{1}$ & $\operatorname{proj}_{1}$ \\
\hline $\operatorname{proj}_{2}$ & $\operatorname{proj}_{2}$ & $\operatorname{proj}_{2}$ & $\operatorname{proj}_{2}$ & $\operatorname{proj}_{2}$ \\
\hline$\neg \circ \operatorname{proj}_{1}$ & $\operatorname{proj}_{1}$ & $\operatorname{proj}_{1}$ & $\operatorname{proj}_{1}$ & $\operatorname{proj}_{1}$ \\
\hline$\neg \circ \mathrm{proj}_{2}$ & $\operatorname{proj}_{2}$ & $\operatorname{proj}_{2}$ & $\operatorname{proj}_{2}$ & $\operatorname{proj}_{2}$ \\
\hline$\wedge$ & const $_{0}$ & $\operatorname{proj}_{1}$ & $\operatorname{proj}_{2}$ & XOR \\
\hline V & XOR & $\operatorname{proj}_{2}$ & $\operatorname{proj}_{1}$ & const $_{0}$ \\
\hline NAND & const $_{0}$ & $\operatorname{proj}_{1}$ & $\operatorname{proj}_{2}$ & XOR \\
\hline NOR & XOR & $\operatorname{proj}_{2}$ & $\operatorname{proj}_{1}$ & const $_{0}$ \\
\hline$\longleftarrow$ & $\operatorname{proj}_{2}$ & XOR & const $_{0}$ & $\operatorname{proj}_{1}$ \\
\hline$\longrightarrow$ & $\operatorname{proj}_{1}$ & const $_{0}$ & XOR & $\operatorname{proj}_{2}$ \\
\hline 4 & $\operatorname{proj}_{2}$ & XOR & const $_{0}$ & $\operatorname{proj}_{1}$ \\
\hline$\nrightarrow$ & $\operatorname{proj}_{1}$ & const $_{0}$ & XOR & $\operatorname{proj}_{2}$ \\
\hline$\longleftrightarrow$ & XOR & XOR & XOR & XOR \\
\hline XOR & XOR & XOR & XOR & XOR \\
\hline
\end{tabular}

Figure 2: Boolean connectives and their hypercube Reed-Akers-Lee-Vichniac differentials.

Likewise, every function from $\mathbf{B}^{2}$ to $\mathbf{B}$ is everywhere differentiable. However, not every function from $\mathbf{B}$ to $\mathbf{B}^{2}$ is differentiable.

To see that Boolean derivatives do not always obey the conclusion of the chain rule, let $t$ be an independent variable, let $x=t$, let $y=t$, and let $z=x \vee y$. Then

$$
\frac{d z}{d t} \neq \frac{\partial z}{\partial x} \frac{d x}{d t}+\frac{\partial z}{\partial y} \frac{d y}{d t}
$$

since

$$
\frac{d z}{d t}=1
$$

whereas

$$
\frac{\partial z}{\partial x} \frac{d x}{d t}+\frac{\partial z}{\partial y} \frac{d y}{d t}=0
$$

What went wrong?

Answer: Two of the hypotheses of Theorem 4.1 do not hold here. Both at $x=0$ and at $x=1$,

$$
D_{x}(\vee \circ(\mathrm{id}, \mathrm{id}))=D_{x} \mathrm{id}=\mathrm{id}
$$

but we cannot assert (indeed, we cannot meaningfully write)

$$
D_{x}(\vee \circ(\mathrm{id}, \mathrm{id}))=\left(D_{(x, x)} \vee\right) \circ\left(D_{x}(\mathrm{id}, \mathrm{id})\right)
$$

since the diagonal embedding (id, id) of $\mathbf{B}$ into $\mathbf{B}^{2}$, although linear, is neither continuous nor differentiable in this calculus. 
We can remedy this failure by equipping $\mathbf{B}^{2}$ with the product convergence structure generated by the convergence structure on $\mathbf{B}$, instead of the hypercube structure used in Example 4.5.

\section{Example 4.6. The Reed-Akers-Lee-Vichniac calculus of Boolean total derivatives} $([38,4,32]$.

As in (Example 4.5), take the finite-dimensional vector spaces $\mathbf{B}^{n}$ as the carriers of objects, take the zero vector of length $n$ as the origin of $\mathbf{B}^{n}$, and take the group generated by the flips $h_{1}, h_{2}, \ldots, h_{n}$ as the translation group of $\mathbf{B}^{n}$,

As before, equip the two-element field $\mathbf{B}$ with the postdiscrete convergence structure induced by taking 0 and 1 as the vertices of a complete digraph.

However, instead of equipping $\mathbf{B}^{n}$ with a hypercube structure, equip $\mathbf{B}^{n}$ with the product convergence structure (which coincides with the postdiscrete convergence structure induced by the complete digraph on $\mathbf{B}^{n}$ ).

As before, for each $m$ and $n$, take the continuous $\mathbf{B}$-linear functions from $\mathbf{B}^{m}$ to $\mathbf{B}^{n}$ as the differentials from $\mathbf{B}^{m}$ to $\mathbf{B}^{n}$.

In general, the set of differentials from $\mathbf{B}^{m}$ to $\mathbf{B}^{n}$ will be larger than before. Every function between complete digraphs is edge-preserving, and therefore every function from $\mathbf{B}^{m}$ to $\mathbf{B}^{n}$ is continuous. In short, the differentials from $\mathbf{B}^{m}$ to $\mathbf{B}^{n}$ are the $\mathbf{B}$-linear functions.

Since the convergence structure on $\mathbf{B}$ and the set of differentials from $\mathbf{B}$ to itself are the same as in Example 4.5, the differentials of functions from $\mathbf{B}$ to itself are exactly the same as in Example 4.5.

Since the underlying sets of objects are the same as before, and there are more differentials, one might perhaps expect differentiability to be more easily satisfied than before, but, in fact, the reverse is true. Differentiability of a function at a point determined by the function's local behavior. Whereas, before, a point in $\mathbf{B}^{n}$ had only $n+1$ out-neighbors (including itself), now it has $2^{n}$ outneighbors. In short, differentiability of a funtion at a point now depends on the function's global behavior.

We leave it to the reader to verify that a function $f: \mathbf{B}^{m} \longrightarrow \mathbf{B}^{n}$ is differentiable at a bit vector $\vec{b}$ if and only if $f$ is an affine function, i.e. if and only if there is a $\mathbf{B}$-linear function $L: \mathbf{B}^{m} \longrightarrow \mathbf{B}^{n}$ such that

$$
f(\vec{x})=L(\vec{x})+f(0) \quad\left(\vec{x} \in \mathbf{B}^{m}\right)
$$

and that, if such an $L$ exists, then $L$ is the unique differential of $f$ everywhere on $\mathbf{B}^{m}$.

Again, let $t$ be an independent variable, let $x=t$, let $y=t$, and let $z=x \vee y$. Since partial derivatives are the same as before, it is still true that

$$
\frac{d z}{d t} \neq \frac{\partial z}{\partial x} \frac{d x}{d t}+\frac{\partial z}{\partial y} \frac{d y}{d t}
$$

What went wrong this time?

Answer: Again, there is a hypothesis of Theorem 4.1 which does not hold. Both at $x=0$ and at $x=1$,

$$
D_{x}(\vee \circ(\mathrm{id}, \mathrm{id}))=D_{x} \mathrm{id}=\mathrm{id}
$$




\begin{tabular}{|c|c|c|c|c|}
\hline \multirow[t]{2}{*}{ Function } & \multicolumn{4}{|c|}{ Differential at } \\
\hline & $(0,0)$ & $(0,1)$ & $(1,0)$ & $(1,1)$ \\
\hline const $_{0}$ & const $_{0}$ & const $_{0}$ & const $_{0}$ & const $_{0}$ \\
\hline const $_{1}$ & const $_{0}$ & const $_{0}$ & const $_{0}$ & const $_{0}$ \\
\hline $\operatorname{proj}_{1}$ & $\operatorname{proj}_{1}$ & $\operatorname{proj}_{1}$ & $\operatorname{proj}_{1}$ & $\operatorname{proj}_{1}$ \\
\hline $\operatorname{proj}_{2}$ & $\operatorname{proj}_{2}$ & $\operatorname{proj}_{2}$ & $\operatorname{proj}_{2}$ & $\operatorname{proj}_{2}$ \\
\hline$\neg \circ$ proj $_{1}$ & $\operatorname{proj}_{1}$ & $\operatorname{proj}_{1}$ & $\operatorname{proj}_{1}$ & $\operatorname{proj}_{1}$ \\
\hline$\neg \circ \mathrm{proj}_{2}$ & $\operatorname{proj}_{2}$ & $\operatorname{proj}_{2}$ & $\operatorname{proj}_{2}$ & $\operatorname{proj}_{2}$ \\
\hline$\wedge$ & & & & \\
\hline V & & & & \\
\hline NAND & & & & \\
\hline NOR & & & & \\
\hline$\longleftarrow$ & & & & \\
\hline$\longrightarrow$ & & & & \\
\hline 4 & & & & \\
\hline$\nrightarrow$ & & & & \\
\hline$\longleftrightarrow$ & XOR & XOR & XOR & XOR \\
\hline XOR & XOR & XOR & XOR & XOR \\
\hline
\end{tabular}

Figure 3: Boolean connectives and their linear Reed-Akers-Lee-Vichniac differentials.

but again, we cannot assert (indeed, cannot meaningfully write)

$$
D_{x}(\vee \circ(\mathrm{id}, \mathrm{id}))=\left(D_{(x, x)} \vee\right) \circ\left(D_{x}(\mathrm{id}, \mathrm{id})\right)
$$

The diagonal embedding (id, id) of $\mathbf{B}$ into $\mathbf{B}^{2}$ is continuous (and, in fact, is a differential) in this calculus, but $\vee$ is nowhere differentiable, although its partials exist everywhere. This should not be astonishing; analogous phenomena exists in the classical multivariable calculus of Newton and Leibniz.

Nevertheless, it is possible to define a Boolean differential calculus with a reliable chain rule, provided that we do not insist that differentials be linear.

\section{Example 4.7. The homogeneous Boolean differential calculus}

Let the objects be precisely the same as in Example 4.6.

For each $m$ and $n$, define the differentials from $\mathbf{B}^{m}$ to $\mathbf{B}^{n}$ to be all origin-preserving functions from $\mathbf{B}^{m}$ to $\mathbf{B}^{n}$. (Equivalently, define the differentials to be the $\mathbf{B}$-homogeneous functions of degree one.) As noted before, this is compatible with our definition of a differential calculus, since every function between complete digraphs is edge-preserving.

Since the convergence structure on $\mathbf{B}$ and the set of differentials from $\mathbf{B}$ to itself are unchanged, the differentials of functions from $\mathbf{B}$ to itself are exactly the same as before. 


\begin{tabular}{|c|c|c|c|c|}
\hline \multirow[t]{2}{*}{ Function } & \multicolumn{4}{|c|}{ Differential at } \\
\hline & $(0,0)$ & $(0,1)$ & $(1,0)$ & $(1,1)$ \\
\hline const $_{0}$ & const $_{0}$ & const $_{0}$ & const $_{0}$ & const $_{0}$ \\
\hline const $_{1}$ & const $_{0}$ & const $_{0}$ & const $_{0}$ & const $_{0}$ \\
\hline $\operatorname{proj}_{1}$ & $\operatorname{proj}_{1}$ & $\operatorname{proj}_{1}$ & $\operatorname{proj}_{1}$ & $\operatorname{proj}_{1}$ \\
\hline $\operatorname{proj}_{2}$ & $\operatorname{proj}_{2}$ & $\operatorname{proj}_{2}$ & $\operatorname{proj}_{2}$ & $\operatorname{proj}_{2}$ \\
\hline$\neg \circ \operatorname{proj}_{1}$ & $\operatorname{proj}_{1}$ & $\operatorname{proj}_{1}$ & $\operatorname{proj}_{1}$ & $\operatorname{proj}_{1}$ \\
\hline$\neg \circ \mathrm{proj}_{2}$ & $\operatorname{proj}_{2}$ & $\operatorname{proj}_{2}$ & $\operatorname{proj}_{2}$ & $\operatorname{proj}_{2}$ \\
\hline$\wedge$ & $\wedge$ & $\nrightarrow$ & 4 & V \\
\hline V & V & 4 & $\nrightarrow$ & $\wedge$ \\
\hline NAND & $\wedge$ & $\nrightarrow$ & 4 & V \\
\hline NOR & V & 4 & $\nrightarrow$ & $\wedge$ \\
\hline$\longleftarrow$ & 4 & V & $\wedge$ & $\longrightarrow$ \\
\hline$\longrightarrow$ & $\nrightarrow$ & $\wedge$ & V & 4 \\
\hline 4 & 4 & V & $\wedge$ & $\nrightarrow$ \\
\hline$\nrightarrow$ & $\nrightarrow$ & $\wedge$ & V & 4 \\
\hline$\longleftrightarrow$ & XOR & XOR & XOR & XOR \\
\hline XOR & XOR & XOR & XOR & XOR \\
\hline
\end{tabular}

Figure 4: Boolean connectives and their homogeneous differentials.

The situation is quite different for functions of more than one variable. We leave it to the reader to verify that every function from $\mathbf{B}^{m}$ to $\mathbf{B}^{n}$ is uniquely differentiable.

Since all functions are continuous and differential, the chain rule (Theorem 4.1) is always applicable in the homogeneous Boolean differential calculus. For instance,

$$
\begin{aligned}
\mathrm{id} & =D_{0} \mathrm{id} \\
& =D_{0}(\vee \circ(\mathrm{id}, \mathrm{id})) \\
& =\left(D_{(0,0)} \vee\right) \circ\left(D_{0}(\mathrm{id}, \mathrm{id})\right) \\
& =\vee \circ(\mathrm{id}, \mathrm{id}) \\
& =\mathrm{id}
\end{aligned}
$$

Likewise,

$$
\begin{aligned}
\text { id } & =D_{1} \text { id } \\
& =D_{1}(\vee \circ(\mathrm{id}, \mathrm{id})) \\
& =\left(D_{(1,1)} \vee\right)\left(D_{1}(\mathrm{id}, \mathrm{id})\right) \\
& =\wedge \circ(\mathrm{id}, \mathrm{id}) \\
& =\mathrm{id}
\end{aligned}
$$




\subsection{Differential Calculi With Nonhomogeneous Objects}

In the preceding development, convergence spaces without full translation groups are "second class citizens" in the sense that they cannot be the carriers of objects of a differential calculus. A somewhat more general (and slightly more complicated) concept of "differential calculus" permits all convergence spaces to be carriers of objects.

Observation 4.2. Let $T$ be a translation group on a convergence space $X$. For each point $x$ of $X$, let $[x]_{T}$ be the $T$ - orbit of $x$, i.e. $[x]_{T}=\{x+\tau \mid \tau \in T\}$.

If $X$ is nonempty, then the set of all $T$ orbits partitions $X$ into homogeneous subspaces. For each $T$-orbit $[x]_{T}$, the restrictions of the members of $T$ to $[x]_{T}$ form a full translation group $T_{[x]}$ on $[x]_{T}$.

Each $T_{[x]}$ is a quotient group of $T$.

Definition 4.4. A system of origins for a convergence space $X$ with respect to a translation group $T$ is a set of representatives of the $T$-orbits, i.e., a subset $\mathcal{O}$ of $X$ containing precisely one member of each $T$-orbit.

For each point $x$ of $X$, let $0_{x}$ be the unique member of $\mathcal{O}$ belonging to the same $T$-orbit as $x$.

Definition 4.5. Let $f: X \longrightarrow Y$ be a function between convergence spaces. Let $\mathcal{O}_{X}\left(\mathcal{O}_{Y}\right.$, respectively) be a system of origins for $X$ with respect to a translation group $S$ (for $Y$ with respect to a translation group $T$, respectively).

i. $f$ will be said to respect orbits iff, for each pair of points $p$ and $q$ of $X$, if $p$ and $q$ lie in the same $S$-orbit, then $f(p)$ and $f(q)$ lie in the same $T$-orbit.

ii. $f$ will be said to be preserve origins iff $f\left(\mathcal{O}_{X}\right) \subseteq \mathcal{O}_{Y}$.

Definition 4.6. A generalized differential calculus is a category $\mathcal{D}$ in which

i. every object of $\mathcal{D}$ is a triple $\mathcal{X}=(X, T, \mathcal{O})$ such that $X$ is a convergence space, $T$ is a translation group on $X$, and $\mathcal{O}$ is a system of origins for $X$ with respect to $T$.

ii. every arrow in $\mathcal{D}$ from an object $\left(X, S, \mathcal{O}_{X}\right)$ to an object $\left(Y, T, \mathcal{O}_{Y}\right)$ is a continuous, orbitrespecting, origin-preserving function from $X$ to $Y$.

iii. composition of arrows in $\mathcal{D}$ is function composition.

iv. for every object $\mathcal{X}=(X, T, \mathcal{O})$, the identity function on $X$ is an arrow in $\mathcal{D}$ from $\mathcal{X}$ to $\mathcal{X}$

v. for each pair of objects $\mathcal{X}=\left(X, S, \mathcal{O}_{X}\right)$ and $\mathcal{Y}=\left(Y, T, \mathcal{O}_{Y}\right)$ and each $\zeta$ in $\mathcal{O}_{Y}$, the constant function mapping every point of $X$ to $\zeta$ is an arrow in $\mathcal{D}$ from $\mathcal{X}$ to $\mathcal{Y}$

A differential calculus (in the sense of Definition 1) is essentially the same notion as a generalized differential calculus in which the translation group of every object is a full translation group.

At the opposite extreme, there are generalized differential calculii in which the translation group of every object is trivial (and hence all orbits are singletons).

Approved for Public Release; Distribution Unlimited. 


\section{Example 4.8. CONV as a generalized differential calculus}

The objects of the trivial generalized differential calculus are all convergence spaces, equipped with trivial translation groups. The arrows from an object $X$ to an object $Y$ are all continuous functions from $X$ to $Y$. (Since all orbits are singletons, every function is orbit-respecting and origin-preserving.)

Let $a \in X$ and let $b \in Y$, where $\mathcal{X}=\left(X, S, \mathcal{O}_{X}\right)$ and $\mathcal{Y}=\left(Y, T, \mathcal{O}_{Y}\right)$ are objects of a generalized differential calculus $\mathcal{D}$. Let $f: A \longrightarrow B$ be an arbitrary function.

Let $L \in \mathcal{D}(\mathcal{X}, \mathcal{Y})$, where, again, $\mathcal{D}(\mathcal{X}, \mathcal{Y})$ is the set of all arrows in $\mathcal{D}$ from $\mathcal{X}$ to $\mathcal{Y}$, equipped with the subspace convergence structure inherited from the function space $Y^{X}$ in CONV.

Definition 4.7. $L$ is a differential of $f$ at $a$ iff for every $\mathcal{F} \downarrow a$ in $X$, there is some $\mathcal{H} \downarrow L$ in $\mathcal{D}(\mathcal{X}, \mathcal{Y})$ such that

i. $\mathcal{H} \subseteq[\{L\}]$, and

ii. for every $H \in \mathcal{H}$, there is some $F \in \mathcal{F}$ such that for every point $x \in F$, there is at least one function $t \in H$ such that

$$
t(x-a)=f(x)-f(a)
$$

Note that $y-a=\tau_{a}^{-1}(y)$.

Differentiability and unique differentiability are defined exactly as before.

\section{Example 4.9. The classical affine differential calculus of real variables}

The objects of this generalized differential calculus are the Euclidean spaces, equipped with trivial translation groups. The arrows from $\mathbf{R}^{m}$ to $\mathbf{R}^{n}$ are all affine functions from $\mathbf{R}^{m}$ to $\mathbf{R}^{n}$

As in the classical linear differential calculus, a function $f$ has a differential at a point $p$ iff $f$ is differentiable (in the usual sense) at $p$.

Let $E_{p} f$ be the differential of $f$ at $p$ in the classical affine differential calculus of real variables. I.e., $E_{p}(f)$ is the affine function which best approximates $f$ in arbitrarily small neighhborhoods of $p$.

Then the differential of $f$ at $p$ in the classical linear differential calculus is the unique linear function which can be obtained from $E_{p} f$ by composing it on both sides with translations (in the usual sense), i.e. the function which maps each point $q$ to $f(p)+\left(E_{p} f\right)(q-p)$

\subsection{The Chain Rule for Generalized Differential Calculii}

Let $a \in X$, where $\mathcal{X}=\left(X, R, \mathcal{O}_{X}\right), \mathcal{Y}=\left(Y, S, \mathcal{O}_{Y}\right)$, and $\mathcal{Z}=\left(Z, T, \mathcal{O}_{Z}\right)$ are objects of a generalized differential calculus $\mathcal{D}$. Let $f: X \longrightarrow Y$ and $g: Y \longrightarrow Z$ be arbitrary functions.

Let $K: \mathcal{X} \longrightarrow \mathcal{Y}$ and $L: \mathcal{Y} \longrightarrow \mathcal{Z}$ be arrows of $\mathcal{D}$

Theorem 4.2. (Chain Rule) Suppose that $f$ is continuous at $a$. Also suppose that $K$ is a differential of $f$ at $a$, and $L$ is a differential of $g$ at $f(a)$.

Then $L \circ K$ is a differential of $g \circ f$ at $a$.

The proof of Theorem 4.2 is identical to the proof of Theorem 4.1, with the obvious modifications.

Approved for Public Release; Distribution Unlimited. 


\subsection{Heterotic Dynamical Systems: Classical and Quantum}

Category-theoretic characterizations of heterotic models of computation were introduced by Stepney et al. in [40]. As remarked above in section 3.1, Heterotic models of computation combine computational models such as classical/quantum, digital/analog, synchronous/asynchronous, imperative/functional/relational, etc. to obtain increased computational power, both practically and theoretically. A simple but highly informative classical/quantum heterotic model of computation is represented by the simple sequential imperative quantum programming language presented by Abramsky in [2].

Abramsky's language, in its simplest form, is built around three types: nat, bit and qubit. The language's commands are of six kinds, the first four of which are: assignment, noop, conditional and loop while. The ability to build up arithmetic expressions of type nat and boolean expressions of type bit is presumed and intended to be sufficient for having a "Turing complete" language. Commands can be sequenced. This fragment of the language is sequential and classical. Finitely branching probabilistic nondeterminism is obtained by adding the command colorfully given by cointoss $p$ in $\mathrm{v}$, where $p$ is an explicit real number in the closed unit interval and $\mathrm{v}$ is of type bit. Operationally, when executed, the cointoss command assigns 0 to $\mathrm{v}$ with probability $p$, and 1 to $\mathrm{V}$ with probability $1-p$. A stock of commands consisting of 1-argument and 2-argument explicit unitary qubit operations that can be applied to variables of type qubit are included. Finally, a quantum measurement command is included: measure $q$ in $v$, where $q$ is of type qubit and $v$ is of type bit.

The states of a qubit variable are wave functions of the classical bit values. A concise way to describe such wave functions is that they are the unit vectors in the (free) complex vector space on the set $\{0,1\}$, where the elements of $\{0,1\}$ are assumed to be an orthonormal basis. We use Dirac notation to denote the elements of this basis by $|0\rangle$ and $|1\rangle$. Thus a state of a qubit variable is given by a vector $a|0\rangle+b|1\rangle$ where $a$ and $b$ are complex numbers satisfying $|a|^{2}+|b|^{2}=1$. Operationally, when measure $\mathrm{q}$ in $\mathrm{v}$ is executed, where the state of $\mathrm{q}$ is $a|0\rangle+b|1\rangle, \mathrm{v}$ is assigned 0 with probability $|a|^{2}$ and is assigned 1 with probability $|b|^{2}$. Suppose that in a particular program $P$, the qubit variables are $\mathrm{q}_{1}, \ldots, \mathrm{q}_{n}$. The states of all of these variables in the aggregate are the unit vectors in the (free) complex vector space on the set of 0,1 -valued $n$-tuples, which is the tensor product of the vector spaces corresponding to the individual qubits, a $2^{n}$-dimensional complex space. The latter presents a complication: take, for example, two qubits $\mathrm{p}$ and $\mathrm{q}$ jointly entangled in the Bell state $\frac{1}{\sqrt{2}}(|0\rangle \otimes|1\rangle+|1\rangle \otimes|0\rangle)$. There are no states of the individual qubits $\mathrm{p}$ and $\mathrm{q}$ whose tensor product is the Bell state. The general state of the two cubits has the form $a_{00}(|0\rangle \otimes|0\rangle)+a_{01}(|0\rangle \otimes|1\rangle)+a_{10}(|1\rangle \otimes|0\rangle)+a_{11}(|1\rangle \otimes|1\rangle)$. Should a measurement of, say p be performed and return the result, say 1 , the state of the pair jumps to $\left(\left|a_{10}\right|^{2}+\left|a_{11}\right|^{2}\right)^{-1 / 2}\left(a_{10}(|1\rangle \otimes\right.$ $\left.|0\rangle)+a_{11}(|1\rangle \otimes|1\rangle)\right)$. Similar jumps happen with measurements of a single qubit among $n$ qubits. For this reason the state of all of the qubit variables must evolve in the aggregate during the course of a computation and explains the approach we take below to the qubit components of the heterotic dynamical systems we employ.

A heterotic dynamical system (greater generality is possible and needed for some applications than we give here) is given by a coupled system of first order differential equations

$$
\left\{\frac{d x_{i}}{d t}=f_{1}\left(x_{1}, \ldots, x_{n}, t\right) \mid i=1, \ldots, n\right\}
$$

where each $x_{i}$ is a function from the reals to a convergence space $X_{i}$. For this system of equations 
to make sense, the derivatives of the $x_{i}$ need to be defined. Furthermore, to capture probabilistic nondeterministic operations such as occur in Abramsky's language, we must have available among the $f_{i}$ "multi-valued functions" that probabilistically return values from a finite set of possibilities [16].

The sequential nature of Abramsky's language is captured in a heterotic dynamical system by the functions $f_{i}$ that can constrain state transitions to occur only at specific times and including an auxiliary variable to serve as a program counter. For assignment commands involving integer arithmetic expressions, the right-hand side of the corresponding differential equation must determine how the value of the variable is to change. To this end, the set of integers $\mathcal{Z}$ is equipped with the convergence structure corresponding to the final segment topology and the space $D(\mathcal{R}, \mathcal{Z})$ of differentials of functions from the reals to the integers is chosen to consist of step functions of a somewhat special kind: for each pair of integers $m$ and $n$, with $m \leq n$, the step functions defined by $g_{t_{0}}(t)=$ if $t<t_{0}$ then $n$ else $m$ and $h_{t_{0}}(t)=$ if $t \leq t_{0}$ then $m$ else $n$. A unitary operation $U$ on quantum registers (i.e. the qubits taken in the aggregate) is implemented by having the right-hand side of the differential equation governing the register produce the unitary operator. Regarding programs in Abramsky's language as heterotic dynamical systems also allows to couple the programs to ordinary real and complex dynamical systems whose states evolve in continuous time.

In classical computation, there are formal verification methods based on specification logic [1]. Formulas in specification logic take the form

$$
\{\Phi\} C\{\Psi\}
$$

where formulas $\Phi$ and $\Psi$ are expressed in an underlying logic (usually first-order predicate logic) and where $C$ is a command; i.e. a program or fragment of a program. Formal semantics are given by the notion that the state map defined by $\mathrm{C}$ maps a computation state about which $\Phi$ is true to a state about which $\Psi$ is true; i.e.

$$
\llbracket\{C\} \rrbracket\{\Phi\} \subseteq\{\Psi\}
$$

where $\llbracket\{C\} \rrbracket$ is the map on computation states defined by the command $C$. The analogous specification formulas for quantum computation can be taken to be

$$
\left\{\text { Trace } \rho P_{\Phi}^{A} \geq p\right\} U\left\{\text { Trace } \rho P_{\Psi}^{A} \geq q\right\}
$$

where $A$ is an observable and $P_{\Delta}^{A}=\chi_{\Delta(A)}$, the characteristic function of effective Borel set $\Delta$. (See [21] for the derivation of the probability that the result of a measurement of observable $A$ with be in $\Delta$ if a system is in a quantum state given by positive definite trace 1 operator $\rho$.) $U$ is a command implementing a unitary transformation

An effective Borel set is defined as follows. Let $P\left(\alpha, x_{1}, \ldots, x_{n}\right)$ be a computable $(1, n)$-ary relation, where the $x_{i}$ are nonnegative integers and $\alpha$ is a function mapping nonnegative integers to nonnegative integers. Computable means that with an oracle for computing $\alpha$, we need only use the oracle to compute some finite sequence $\alpha(0), \alpha(1), \ldots, \alpha(k)$. to then effectively decide whether or not $P\left(\alpha, x_{1}, \ldots, x_{n}\right)$ is true. Consider

$$
\forall x_{1} \exists x_{2} \cdots Q x_{n} P\left(\alpha, x_{1}, \ldots, x_{n}\right)
$$

where $Q$ is either $\forall$ or $\exists$ in the alternating quantifier prefix in the above expression. In the conventional notation widely used in mathematical logic, the sets of all $\alpha$ satisfying formulas such as the 
above are the $(1,0)$-ary arithmetic relations when interpreted on the natural numbers or any other effectively presented countably infinite set such as the integers. These sets are all Borel and the set of their characteristic functions are the Borel sets of configurations we have been discussing. In a set-theoretic sense these constitute a countable subcollection of the uncountably many distinct Borel sets of configurations we have been implicitly considering, but they include all Borel sets of configurations that can be explicitly defined via the apparatus of formal number theory.

\section{CONCLUSIONS}

The investigations discussed in this report began with the goal of developing persistent homology to be a new, additional tool for software validation. After an initial exploration of the utility of persistent homology for detection of dynamic geometric structure in the deformations of low dimensional manifolds, this phase of the project concluded that applications of persistent homology to high dimensional complicated dynamical systems is premature $[5,15]$. The project then focused on developing the mathematically rigorous specification and formal semantics of software involving variables with compound type and that potentially aggregates more than one model of computation, which we modeled by heterotic dynamical systems. This focus on rigorous formal semantics of programs for complex cyber systems was judged to be necessary for developing formal validation methods. The capabilities developed during this investigation have provided a way forward to the following milestones:

1. Development of a specification logic for verification of closely coupled classical and quantum computational processes.

2. Development of integration on convergence spaces for rigorous reasoning about the computation of the trajectories of coupled digital/analog heterotic systems.

3. Enhancement of formal methods for synthesizing programs for adiabatic quantum computation.

\section{Acknowledgements}

Contractor acknowledges Government's support in the publication of this report. We acknowledge all co-authors and colleagues that contributed to this research. This material is based upon work funded by AFRL, under AFRL Contract No. FA8750-11-2-0275. Any opinions, findings and conclusions or recommendations expressed in this material are those of the author and do not necessarily reflect the views of AFRL. 


\section{REFERENCES}

[1] "Domain Theory," in Handbook of Logic in Computer Science, vol 3, S. Abramsky, Dov M. Gabbay, and T. S. E. Maibaum (eds.), (Clarendon Press, Oxford. 1994).

[2] S. Abramsky, “A cooks tour of a simple quantum programming language." In 3rd International Symposium on Domain Theory, (2004).

[3] J. Adámek, H. Herrlich, and G. E. Strecker, Abstract and Concrete Categories, (Wiley Interscience, 1990).

[4] S. B. Akers Jr., "On a theory of Boolean functions”, J. SIAM, 7, 4 (1959), 487-498.

[5] Alsing. P., Blair, H. A., Corne, M., Jones, G., Miller, W. A., Mischaikow, K. and Nanda, V. "Topological Signatures of Singularities in Simplicial Ricci Flow," to be submitted to J. Comp. Geom. (2014).

[6] M. A. Arbib and E. Manes, Arrows, Structures, and Functors: The categorical imperative, (Academic Press, 1975).

[7] R. F. Arens, "A Topology for spaces of transformations". Annals of Mathematics, 247 (1946), 480-495.

[8] W. I. Averbukh and O. G. Smolyanov, "The various definitions of the derivative in linear topological spaces”, Russian Math. Surveys, 234 (1968), 67-113.

[9] F. Bagnoli, "Boolean derivatives and computation of cellular automata", Int. J. Modern Physics C, 3 (1992) 309-320.

[10] R. Beattie, R. and H.-P. Butzmann, Convergence structures and Applications to Functional Analysis, (Kluwer Academic Publishers, Dordrecht, 2003).

[11] E. Binz, "Ein Differenzierbarkeitsbegriff limitieren Vektorraäume", Comment. Math. Helv., 41, (1966), 137-156.

[12] E. Binz and E. Keller, "Functionenräume in der Kategorie der Limesräume", Ann. Acad. Sci. Fenn., Ser. A.I., (1966), 1-21.

[13] E. Binz, Continuous Convergence on C(X), Lecture Notes in Math. 469, (Springer-Verlag, 1966).

[14] H.A. Blair, D.W. Jakel, R.J. Irwin and A. Rivera, "Elementary differential calculus on discrete and hybrid structures", Logical Foundations of Computer Science, S. N. Artemov and A. Nerode, editors, (Lecture Notes in Computer Science, 4514, Springer-Verlag, 2007).

[15] H.A. Blair, J.C. Bush, M.A. Corne, X. Gu, J.R. McDonald and W.A. Miller, "Geometric and topological tools for analysis of cyber Systems", Poster, NEXT Conference on Technology, Manufacturing and Innovation. 
[16] D.R. Patten, H.A. Blair and P.M. Alsing, "Heterotic quantum dynamical systems: an application of differential calculus on digraphs", 14th Haifa Workshop on Interdisciplinary Applicationsof Graph Theory, Combinatorics and Algorithms, Haifa, Israel, (2014)

[17] G. Bordaud, "Some cartesian closed categories of convergence spaces", Convergence Spaces: Proc. Conf. Mannheim 1975, (Lecture Notes in Mathematics, Springer-Verlag, 540, 1979), 93-98.

[18] N. Bourbaki, Topologie Générale, Actualités Sci. Ind. 858 (1940), 916 (1942), 1029 (1947), 1045 (1948), 1084 (1949).

[19] C. Choquet, “Convergences”, Ann. Univ. Grenoble, 23, (1947), 55-112.

[20] J. Dugundji, Topology, (Allyn and Bacon, 1966).

[21] G. Fano, Mathematical Methods of Quantum Mechanics. (McGraw-Hill, 1971.)

[22] R. H. Fox, “On topologies for function spaces”, Bull. Amer. Math. Soc., 51, (1945), 429432.

[23] A. Frölicher and W. Bucher, "Calculus in Vector Spaces without Norm", Lecture Notes in Math., 30, Springer-Verlag, (1966).

[24] R. Heckmann, “A non-topological view of dcpo's as convergence spaces”, Theoretical Computer Science, 305 (2003) 159-186.

[25] H. Herrlich, Topologische Reflexionen und Coreflexionen, (Lecture Notes Math. 78, Springer-Verlag, 1968).

[26] W. Imrich and S. Klavžar, Product Graphs, (John Wiley and Sons, 2000).

[27] M. Katĕtov, "On continuity structures and spaces of mappings", Comm. Math. Univ. Carol. 62 (1965), 257-279.

[28] E. Keller, "Differential calculus in locally convex spaces, Lecture Notes in Math. 417, Springer-Verlag, (1974).

[29] J. L. Kelley, General Topology, (Van Nostrand Reinhold, 1955).

[30] D. C. Kent, "Convergence functions and their related topologies", Fund. Math. 54, (1964), 125-133.

[31] A. Kriegl, "Eine kartesische abgeschlossene Kategorie glatter Abbildungen zwischen beleibigen lokalkonvexen Vektoräumen”, Monatsh. Math., 95, (1983), 287-309.

[32] S.C. Lee, "Vector Boolean algebra and vector Boolean calculus: theory and applications", in Computer Science and Multiple-Valued Logic: Theory and applications, (D. C. Rine, ed. North-Holland, 1977), 308-338.

[33] S. Mac Lane, Categories for the Working Mathematician, (Graduate Texts in Mathematics, Springer-Verlag, 1971).

Approved for Public Release; Distribution Unlimited. 
[34] G. Marinescu, Espaces Vectoriels Pseudo Topologique et le Théorie de Distributions, (Deutcshe Verlag d. Wiss.), 1963.

[35] A. D. Michal, "Differential calculus in linear topological spaces”, Proc. Nat. Acad. Sci., 24, 8, (1938), 340-342.

[36] D.R. Patten, Problems in the Theory of Convergence Spaces, (Ph.D. Dissertation, Syracuse University College of Engineering and Science, 2014).

[37] D.R. Patten, H.A. Blair and D.W. Jakel,"Differential calculus on finite groups”, (submitted).

[38] I. S. Reed, "A class of multiple-error-correcting codes and the de- coding scheme". IRE Trans. Inform. Theory, IT-4, 9 (1954), 38-49.

[39] C. M. Reidys and P. F. Stadler, Combinatorial landscapes, http://www.santafe.edu/sfi/publications/Working-Papers/01-03-014.pdf

[40] S. Stepney, V. Kendon, P. Hines, and A. Sebald, "A framework for heterotic computing", In 8th International Workshop on Quantum Physics and Logic (QP\&L 2011), (2012) 263273.

[41] B. M. R. Stadler, P. F. Stadler, G. P. Wagner, and W. Fontana, "The topology of the possible: Formal spaces underlying patterns of evolutionary change", Journal of Theoretical Biology, 213, 2 (2001), 241-274.

[42] P. Urysohn, “Über die Machtigkeit der susammenhängen Mengen,” Math. Ann. 94 (1925) 262-295.

[43] G. Y. Vichniac, "Boolean derivatives on cellular automata", in Cellular automata: theory and experiment, (H. Gutowitz, ed., MIT Press, 1991), Physica D, 45, 1-3, (1990), 63-74. 


\section{LIST OF SYMBOLS, ABBREVIATIONS AND ACRONYMS}

AFRL

CONV

$\mathrm{CS}$

PI

PostDISCRETE The category of post discrete convergence spaces

PreTOP The category of pretopological spaces

RDIGRAPH The category of digraphs with self-loops

RITA

Air Force Research Laboratory

The Cartesian closed category of convergence spaces

Convergence Structure

Principle Investigator

Emerging Computing Architectures 